\title{
ACTITUDES DE LOS PADRES HACIA EL RETARDO MENTAL LEVE DE SUS HIJOS EN UNA MUESTRA DE LA PROVINCIA CONSTITUCIONAL DEL CALLAO.
}

\author{
Mg. Juan Pequeña Constantino.
}

Se evalúan las actitudes de los padres hacia los hijos con Retardo Mental a través de un estudio descriptivo comparativo.

La muestra estuvo conformada por los padres y las madres de 54 niños con diagnostico de Retardo Mental seleccionados de los Centros Educativos de Educación Especial del Callao, para ello se utilizó como instrumento una Escala tipo Likert de Actitudes de la madre hacia el hijo con Retardo Mental, tanto en su versión para el hijo como para la hija. Los resultados indicaron que no se alcanzaron diferencias significativas entre los padres y las madres en cuanto a las actitudes generales, y en las áreas de Cuidado Protección, Percepción y Aceptación del Problema Prejuicios, Disciplina, Relaciones Interpersonales, Expectativas de Logro e Integración Familiar, según el sexo del hijo por separado. Tampoco se alcanzaron diferencias significativas al comparar solo en los padres sus actitudes hacia el hijo y la hija y solo en las madres dichas actitudes.

Se concluye que los padres y las madres tienen actitudes similares frente a su hijo con retardo mental.

Finalmente, se recomienda realizar estudios que consideren los diferentes status socioeconómicos con la finalidad de poder generalizar la información.

PALABRAS CLAVE: Actitudes, retardo mental, actitudes padres.

The attitudes of the parents are evaluated towards the children with Mental Retardation, through a comparative descriptive study. The sample was conformed by the parents, of 54 children with I diagnose of Mental Retardation selected of the Educative Centers of Special Education of the Callao, for it was used like instrument a Scale type Likert de Actitudes of the mother towards the son with Mental Retardation, as much in its version for the son Iike for the daughter. The results indicated that significant differences between the parents and the mothers as far as the general attitudes were not reached, and in the areas of Welltaken care of Protection, Perception and Acceptance of the Problem Interpersonal Prejudices, Discipline, Relations, Expectations of Profit and Familiar Integration, according to the sex of the son separately. Significant differences were not reached either when single comparing in the parents their attitudes towards the son and the single daughter and in the mothers these attitudes. One concludes that the parents and the mothers have attitudes similar front to their son with mental retardation. Finally, it is recomme ded to make studies that consider the different socioeconomic status with the purpose of being able to generalize the information.

KEY WORDS: Attitudes, retardation, attitudes parents. 


\section{INTRODUCCIÓN}

El análisis de la experiencia realizada en el trabajo con familias, permite observar de forma empírica una amplia gama de actitudes que presentan los padres hacia su hijo con Retardo mental; actitudes que podían variar de manera notable fluctuando desde una actitud de pasividad y conformismo hasta la de una gran sobreprotección al niño con Retardo mental. La evaluación de estos aspectos nos llevó a considerar que es importante no solo establecer cognitivamente el número de actitudes localizables y el grado de intensidad de las mismas sino además es necesario examinar las variaciones de dichas actitudes que presentan los padres en función al sexo del hijo.

Todo esto fue el elemento motivador que nos impulso a realizar la presente investigación con la finalidad de tener una visión científica de las actitudes que presentan los padres, lo que redundaría en beneficio del niño retardado mental, llevando a plantearnos la siguiente interrogante: ¿Las actitudes de los padres y las madres frente al hijo con Retardo mental Leve difieren significativamente en relación al sexo del hijo con retardo mental leve, en una muestra de familias de condición socioeconómica media baja, con residencia en la Provincia Constitucional del Callao?

\section{Actitudes frente al Retardo Mental}

La historia de las actitudes hacia un determinado objeto social está marcada por los sucesos presentes o pasados en relación con ese objeto; de allí que sea razonable afirmar que las actitudes hacia el retardo mental se encuentran condicionadas por el modo como la sociedad enfrenta el problema en relación al Retardo Mental.

Antes del siglo XIX, el problema no existía científicamente. La fortuna o desdicha del retardo mental inicialmente estuvo guiada por la superstición, explotados o perseguidos y a veces por su conducta extraña, se les atribuía poderes sobrenaturales. Fue en 1918, que Esquirol creó el término «idiocia» y sostuvo que el Retardo mental no era una enfermedad, sino un estado de no desarrollo de las funciones intelectuales, comprobable desde la más tierna edad y que nada lo podía aliviar, tenía que distinguirse dicho estado de la demencia que representaba un deterioro a partir de un desarrollo intelectual normal. Cabe destacar en la definición que da Esquirol dos aspectos esenciales: el origen del déficit intelectual y la nula posibilidad de recuperarse.

Jean Itard, que tuvo a su cargo la educación de un «niño salvaje» de aproximadamente doce años de edad, encontrando en las selvas de Aveyron, sostuvo, que el fracaso del niño en adquirir el lenguaje oral se debía quizás a que el periodo sensible de este aprendizaje ya había pasado irremediablemente.

De 1800 a 1870, surgen acciones humanitarias aisladas, generalmente de líderes religiosos, como también ensayos terapéuticos y educativos. Pero contradictoriamente a estos hechos, en 1840 Morel citado por Pichot (1948) propuso una teoría de la degeneración, según la cual en ciertas familias, a través de las distintas generaciones se produce una degradación progresiva del ser humano llegándose al final del proceso, en la última generación a la idiocia. Esta teoría, representa las ideas y actitudes de la sociedad del siglo XIX, e influyen en muchas de las diferentes concepciones psiquiátricas de la época.

Es a finales del siglo XIX y a principios del siglo XX, que se crean instituciones consagradas a los deficientes medios y profundos. Las instituciones se sobrecargan cada vez más y según Perrón (1968) se convierte en «un poco hospital, un poco cuartel, un poco prisión, el asilo tiene muy poco de escuela».

Esta situación, es consecuencia lógica de la ignorancia que existía con respecto a la deficiencia mental. Aún en la primera década del siglo XX, persisten las actitudes de rechazo hacia el retardo mental. 
En 1 912, Goddard realizó un estudio genealógico de dos familias del este de los Estados Unidos. Esas familias tenían como antepasado a Martín Kalli Kak. La genealogía de los Kalli Kak sirvió de modelo a otras investigaciones históricas psicológicas, a principios del siglo XX, las que destacaban que la herencia era la causa esencial del retardo mental y que solo un pequeño porcentaje de casos eran debido a enfermedades o accidentes.

Por su parte Zigler y Barter (1969) sostuvieron que los primeros años del presente siglo, el punto e vista que se había hecho popular acerca de los individuos intelectualmente fue el señalarlos como esencialmente inmorales, degenerados y depravados. Siendo necesario además destacar que paralelamente a estos sentimientos y opiniones adversas sobre el Retardo mental se producen avances técnicos que permiten evaluar y clasificar a los retardados mentales según el nivel de funcionamiento intelectual. Es en este sentido que Binet y Simon en 1905 y Terman 1960 son los que mayores contribuciones hicieron al respecto. La aparición de la Escala de Binet Simún, permitió de modo provisional e imperfecto definir el retardo mental en términos de coeficiente intelectuales y de la dispersión del grupo.

Algunos años más tarde, se incrementan los conocimientos médicos acerca de los aspectos orgánicos; los psicólogos utilizan pruebas de personalidad para evaluar el ajuste social y la conducta, se realizan nuevos descubrimientos científicos sobre factores genéticos y bioquímicos que amplían los conocimientos con respecto al factor etiológicos del retardo mental a pesar de este cambio en la competencia y del adelanto técnico para su diagnóstico, todavía a mediados del presente siglo algunos autores mantienen un pensamiento cliché y estereotipado sobre el retardo mental.

\section{Investigaciones antecedentes}

Entre los estudios realizados con relación a las actitudes hacia los niños con retardo mental, encontramos que Grebler (1951 1952) comparó las actitudes de los padres hacia el hijo con retardo mental con los de los niños normales. Se observo que los sentimientos de los padres de los niños retardados están expuestos a una mayor frustración debido a la condición inherente del niño y a las limitaciones impuestas por el ambiente. Por su parte Evert y Meredith (1957) investigaron la apreciación de la madre acerca del rendimiento intelectual de su hijo. Mientras Rasen (1959) realizó un estudio exploratorio para sistematizar aspectos seleccionados en el desarrollo de la comprensión de la madre hacia el hijo con retardo mental: toma de conciencia, reconocimiento, búsqueda de causa, búsqueda de solución aceptación y aspectos relacionados con la educación.

Por otro lado Lewis Klebanoff (1959) efectuó una investigación para determinar las actitudes paren tales de las madres de niños con daño cerebral y retardados; esquizofrenia y normales.

Nicholas (1962) llevo a cabo una investigación relacionada con los factores vinculados a las actitudes de los padres. Lave (1967) condujo un estudio para descubrir algunas características relacionadas con las actitudes positivas y negativas de los padres hacia los niños retardados mentales.

Rutter y colaboradores (1970) llevaron adelante una investigación para establecer los efectos del niño limitado en la familia, tomando en cuenta factores tales como la aceptación o rechazo por parte de los padres, la sobreprotección, expectativas para el futuro, la repercusión de este hijo en el núcleo familiar, etc.

Finalmente, Cummings (1976) investigó sobre el impacto de la deficiencia en los padres de niños mentalmente retardados y enfermos crónicos.

En lo que concierne al estudio de este tipo de actitudes en el Perú, la bibliografía reporta que fue Sánchez Otero (1968) quien realizó en primer estudio de actitudes de los padres frente al problema del retraso mental, trabajando con 18 grupos de familiares co- 
rrespondiente a igual número de retrasados mentales de la escuela Especial «Carlos A. Manucci» de la ciudad de Trujillo.

Ayllon (1980) comparó las actitudes de las madres hacia niños retardados mentales educables y entrenables, encontrándose entre los resultados mas irrelevantes que no existían diferencias estadísticamente significativas entre ambos tipos de niños.

Por su parte, Hermoza (1985) comparó las actitudes de un grupo de madres de niños Fronterizos con otro grupo cuyos hijos presentan retardo Retar10 mental Leve. Y finalmente fue Ugarriza (1987) quien utilizó el método de análisis de caminos (Path Analysis) para estudiar las actitudes de las madres hacia el hijo retardado mental.

\section{OBJETIVOS}

Para la ejecución del presente estudio se plantearon los siguientes objetivos principales:

- Describir, comparar y establecer las diferencias de las actitudes de los padres frente a las madres que tienen hijos con retardo mental leve.

- Describir y comparar las actitudes de los dos grupos de padres en las áreas de: cuidado y Protección, Percepción y Aceptación del Problema, Relaciones Interpersonales, Expectativas de Logro, Disciplina, Prejuicios, Integración Familiar.

- Describir las actitudes de los padres considerando el sexo de los hijos con Retardo metal leve.

\section{HIPÓTESIS}

Las hipótesis plantadas fueron las siguientes:

H 1 «Las actitudes de los padres y las madres hacia el cuidado y protección del hijo con Retardo mental Leve difieren significativamente en función al sexo del hijo con dicho retardo».

H2 «Las actitudes de los padres y las madres hacia la Percepción y aceptación del hijo con Retardo mental Leve difieren significativamente en función al sexo del hijo con dicho retardo».

H3 «Las actitudes de los padres y las madres hacia el área de Relaciones interpersonales difieren significativamente en función al sexo del hijo con retardo mental leve».

H4 «Las actitudes de los padres y las madres hacia el área de Expectativas de logro difieren significativamente en función al sexo del hijo con Retardo mental leve».

H5 «Las actitudes de los padres y las madres hacia el área de Disciplina difieren significativamente en función al sexo del hijo con Retardo mental leve».

H6 «Las actitudes de los padres y las madres hacia el área de Prejuicios difieren significativamente en función al sexo del hijo con Retardo metal leve».

H7 «Las actitudes de los padres y las madres hacia el área de Integración familiar difieren significativamente en función al sexo del hijo con Retardo mental leve». 


\section{METODOLOGÍA}

Corresponde a la definición de un estudio descriptivo comparativo en la medida que los procedimientos que se han seguido consisten en organizar la información producto de la aplicación de la escala de «Actitudes de la madre hacia el hijo con retardo mental» y contrastar las actitudes medidas de los padres en función al sexo del hijo.

\section{Variables}

$V$ Independiente. - Ser padre o madre de un hijo con Retardo mental leve niña y/o varón. $V$ Dependiente. - Actitudes que tiene los padres hacia el hijo con retardo mental leve niña y/o varón. Las actitudes a ser estudiadas son:

- Cuidado y Protección (c. P.), atención a las necesidades del niño.

- Percepción y Aceptación del problema (P.A.), se define como la caracterización y aceptación realista del problema diagnosticado como Retardo mental.

- Relaciones Interpersonales (R.L), Intercambios afectivos a través de la comunicación verbal y el contacto físico en la relación padre a niño.

- Disciplina (D), Se entiende como el control de la conducta relacionada con el cumplimiento de normas de juego, la comida, el sueño, los hábitos de limpieza, etc.

- Prejuicio (P).- Actitud hostil o esquivamente benévola hacia el hijo deficiente, simplemente porque pertenece al grupo de retardados mentales y suponiéndose por lo tanto que posee las cualidades objetables atribuidas al grupo.

- Integración Familiar (LE), Referido a como repercute la presencia del niño retardado mental en la unidad familiar.

V. Controlada.- Nivel socioeconómico medio bajo de los padres, conformada por aquellos padres que en una ficha de 9 indicadores socioeconómicos se ubican entre los límites de la media aritmética menos media desviación estándar hasta media aritmética menos una desviación estándar.

\section{Sujetos}

La población estuvo conformada por los niños con retardo mental leve de la provincia constitucional del Callao. Para obtener la muestra se utilizó el método probabilístico intencional manteniendo los siguientes criterios: a) Los niños debían tener un diagnóstico de retardo mental leve; b) tener entre 8 a J3 años de edad; c) ser hijos de familias completas y d) los padres debían tener indicadores definidos de nivel socioeconómico medio bajo.

La muestra estuvo conformada por 54 niños en total (39 niños y 15 niñas) procedentes de los centros de Educación Especial «San Antonio», «La Perla» y «Carmen de la Legua».

Instrumentos de investigación: La Escala de Actitudes de la Madre hacia el Hijo con Retardo Mental Leve.

La escala fue elaborada por Ugarriza y Tapia (Ugarriza 1986) con el propósito fundamental de contar con un instrumento estandarizado válido y confiable que permitiese evaluar las actitudes maternas hacia niños con déficit intelectual. El instrumento corresponde a una escala del tipo Likert, que estuvo integrada por un conjunto de proposiciones o juicios ante los cuales se solicita la reacción de la madre y consta de 56 items o reactivos distribuidos en las siguientes áreas: a) Cuidado y Protección, b) Percepción y Aceptación del problema; c) Relaciones Interpersonales; d) Expectativas de Logro; e) Disciplina; f) Prejuicio; g) Integración Familiar.

Las respuestas a cada Ítem son solicitadas en términos de grados y bajo la forma de alternativas: 1) Totalmente de acuerdo (TA); 2) De acuerdo (DA); 3) Indeciso (1); 4) En desacuerdo (ED); 5) Totalmente en desacuerdo (TD).

La calificación de cada respuesta varia de 1 a 5 y de 5 al, en función del contenido del Ítem sea positivo o negativo respectivamente. El puntaje total de cada persona resulta de 
sumar las puntuaciones alcanzadas en las respuestas a cada Ítem; el puntaje máximo que se puede alcanzar es de 280 y el mínimo de 56 . Los puntajes altos significan una «actitud de aceptación»y los puntajes bajos una «actitud de rechazo».

En el presente estudio se hizo una revisión de los ítems con el propósito de complementar el análisis efectuado por las autoras y sobre esta base seleccionar los que resultan clasificatoriamente pertinentes. Los ítems que no discriminan adecuadamente y que fueron retirados para el presente estudio fueron: a) Para el área de Cuidado y Protección los ítems 041, 051, 071, 081; b) Para el área de Percepción y Aceptación del problema, el Ítem 012; quedando la prueba total de 56 ítems en 51 ítems.

Validez de la escala.- Tapia y Ugarriza (Ugarriza, 1987) indican que desarrollaron el estudio de la validez de contenido realizando el análisis del contenido de los items que conforman la escala, con el propósito de apreciar su grado de representatividad respecto del universo de contenido del cual forma parte. La validación lógica se realizó en base a los supuestos que guiaron la elección de los items. Además, se recurrió a algunos jueces para efectuar la misma tarea, guiados por algunas especificaciones dadas por las autoras de la escala.

La interrogante fundamental que sirvió de guía a este tipo de validación fue evaluar si el Ítem pertenecía al Universo de contenido estudiado.

En el presente estudio se hizo una revisión de los items con el propósito de complementar el análisis efectuado por las autoras y sobre esta base seleccionar los que resulten clasificatoriamente pertinentes.

Confiabilidad de la escala.- Se estudio la consistencia interna de la escala, la cual fue determinada mediante el método de la correlación Ítem-escala y con ello también se pudo determinar el poder discriminativo de cada Ítem. Para este objeto se estableció la correlación entre el puntaje alcanzado en cada Ítem por cada persona y el valor escalar total de la misma (para el cálculo se empleó también el coeficiente de Pearson). Adicionalmente se ha revisado la confiabilidad de la escala mediante el análisis de consistencia interna subtest total habiéndose encontrado coeficientes que van desde un mínimo de 0.54 hasta un máximo de 0.84 ,en todas ellas significativas, dejando entrever que cada una de las escalas son partes constitutivas de las mismas.

\section{Recolección de datos}

De un total de 4 centros educativos especiales estatales de la provincia constitucional del Callao se procedió a seleccionar la muestra quedando eliminado el C.E.E. «Polivalente» por considerarse no significativa la muestra.

De los 202 niños clasificados con el diagnostico de retardo mental leve se procedió a seleccionar intencionalmente con los criterios establecidos para dicha investigación quedando fijado el tamaño muestral en 54 niños procedentes de los C.E.E. «San Antonio», «La Perla»y «Carmen de la Legua».

Se citó a los padres de los niños seleccionados durante las mañanas en distintos horarios, con la finalidad de administrarles el cuestionario de datos personales y la Escala de actitudes de la madre hacia el hijo con Retardo Mental, a un total de 54 padres y 54 madres.

Con los padres que no asistieron a la entrevista se coordinó una entrevista domiciliaria con el propósito de aplicarles dichos cuestionarios. Se tuvo especial cuidado que el cuestionario fuese contestado en su totalidad. 


\section{RESULTADOS}

Los resultados de la investigación nos demuestran que las actitudes generales de los padres en función al sexo de los hijos no difieren aún cuando las actitudes del padre se presentan como positivas tanto hacia el hijo como la hija, mientras que en las madres las actitudes se presentan en forma positiva hacia la hija y ambivalente hacia el hijo, lo que implica que una vez que los padres han aceptado el hecho de tener un hijo con retardo mental es capaz de percibir con toda precisión las aptitudes del niño, su potencial de habilitación, estando plenamente conscientes tanto de sus habilidades como de sus recursos.

En cuanto al cuidado y la protección de los padres no difieren de las madres en sus actitudes tanto del hijo como de la hija, lo que estaría implicando que los padres que aceptan a su hijo retardado serían capaces de brindarle afecto al igual que los otros hijos.

En lo que se refiere a la Percepción y aceptación del problema igualmente no se encuentran diferencias significativas. Lo que demuestra que un adecuada percepción y aceptación de las limitaciones del hijo tienen efectos positivos sobre el modo como lo cuide y proteja, sobre la manera como ejerza controles satisfactorios sobre él y tenga las expectativas realistas sobre sus logros y habilidades. Y por último que admita que no perjudica la integración familiar.

En cuanto a las relaciones interpersonales los padres no difieren en sus actitudes de las madres tanto del hijo como de la hija lo que implica que las actitudes frente a los intercambios afectivos ya sea bajo la forma de expresión verbal o física va a depender directamente de que los padres acepten a su hijo retardado y el clima emocional que reine en la familia.

En general, las expectativas respecto al futuro de sus hijos son realistas en la mayoría de los casos, son pocos los padres que se forman expectativas que están por encima de la verdadera capacidad de su hijo.

No existe diferencia significativa en lo que se refiere a la Disciplina. Lo que implicaría que el niño con retardo es capaz de adaptarse a las exigencias del medio ambiente fomentando una actitud de adaptación frente a la realidad.

En relación a los Prejuicios que tienen los padres de sujetos retardados respecto al problema de su hijo no encontramos diferencias en las actitudes de los padres y las madres. Lo que implicaría que los padres no aceptan como ciertas determinadas creencias que carecen de fundamente y que no se dejen impresionar tratando de hallar soluciones irreales al problema de su hijo cayendo fácilmente en manos de curanderos y seudoprofesionales.

En lo que respecta a la Integración familiar no se observa diferencias en las actitudes de los padres y las madres. Lo que nos demuestra que los padres consideran que la presencia de un niño con retardo mental en el hogar no es una carga para la economía familiar, ni tampoco es un miembro conflictivo que da lugar a situaciones de tensión que no permiten alcanzar la felicidad en el hogar. 


\begin{tabular}{|c|c|c|c|c|c|c|}
\hline ACTITUD & GRUPO & N & $\bar{X}$ & $D S$ & $t$ & $P$ \\
\hline$A C T I T U D D E L$ PADRE & $\begin{array}{l}H D O O \\
H D Q A\end{array}$ & $\begin{array}{l}39 \\
15\end{array}$ & $\begin{array}{l}181.82 \\
185.53\end{array}$ & $\begin{array}{l}17.49 \\
20.13\end{array}$ & .0 .62 & .268 \\
\hline $\begin{array}{c}A C T T V D D E \text { DA } \\
\text { MADRE }\end{array}$ & $\begin{array}{l}H X O O \\
H I I A\end{array}$ & $\begin{array}{l}39 \\
15\end{array}$ & $\begin{array}{l}177.89 \\
182.86\end{array}$ & $\begin{array}{l}17.15 \\
34.78\end{array}$ & .0 .52 & .302 \\
\hline $\begin{array}{c}\text { ACTITUD HACZA EL } \\
\text { HIJO }\end{array}$ & $\begin{array}{l}H X O O \\
F E O A\end{array}$ & $\begin{array}{l}39 \\
15\end{array}$ & $\begin{array}{l}181.82 \\
177.89\end{array}$ & $\begin{array}{l}17.49 \\
17.15\end{array}$ & 200 & .84 \\
\hline $\begin{array}{c}\text { ACTITUD HACZA LA } \\
\text { HIJA }\end{array}$ & $\begin{array}{l}\text { HDO } \\
\text { HDEA }\end{array}$ & $\begin{array}{l}39 \\
15\end{array}$ & $\begin{array}{l}185.53 \\
182.86\end{array}$ & $\begin{array}{l}20.73 \\
94.78\end{array}$ & 0.25 & .599 \\
\hline
\end{tabular}

(*) Significativo $\mathrm{P}<0 \mathrm{OS}$

( ) No significativo.

Cuadro $\mathrm{N}^{\circ}$ 2: Prueba t de student de la actitud por área del padre frente al hijo y la hija

\begin{tabular}{|c|c|c|c|c|c|c|}
\hline AREA & GRUPO & N & $\bar{X}$ & $D S$ & $t$ & $P$ \\
\hline CUIDADO Y PROTECCIÓN & $\begin{array}{l}H W O D \\
H W Z A\end{array}$ & $\begin{array}{l}39 \\
\text { is }\end{array}$ & $\begin{array}{l}18.71 \\
19.13\end{array}$ & $\begin{array}{l}4.04 \\
4.02\end{array}$ & -0.39 & .367 \\
\hline \multirow{2}{*}{$\begin{array}{c}\text { PERCEPCIOW Y ACEPTA DEL } \\
\text { PROBLEMA }\end{array}$} & $H W O$ & 39 & 3256 & 345 & \multirow{2}{*}{-0.87} & \multirow[b]{2}{*}{.192} \\
\hline & $\mathrm{HZZA}$ & ES & 32.40 & 311 & & \\
\hline $\begin{array}{c}\text { RELACIONES } \\
\text { INTERPERSOAVALES }\end{array}$ & FEOO & 39 & 20.66 & 2.72 & .0 .35 & 363 \\
\hline \multirow{2}{*}{ EXPECTATIVAS DE LOGRO } & $\begin{array}{l}\text { BOEA } \\
H W O\end{array}$ & $\begin{array}{l}15 \\
39\end{array}$ & $\begin{array}{l}20.93 \\
28.79\end{array}$ & $\begin{array}{l}2.46 \\
4.33\end{array}$ & \multirow[b]{2}{*}{0.50} & \multirow[b]{2}{*}{.309} \\
\hline & $\mathrm{HOHA}$ & IS & 28. 13 & 4.36 & & \\
\hline \multirow{2}{*}{ DISCIPLINA } & $\mathrm{HWO}$ & 39 & 12.58 & 2.98 & \multirow{2}{*}{.0 .79} & \multirow{2}{*}{.255} \\
\hline & $\mathrm{HOA}$ & IS & 13.26 & 2.66 & & \\
\hline PREJUICIOS & $\begin{array}{l}\mathrm{HWO} \\
\mathrm{HWO}\end{array}$ & ijg & $\begin{array}{l}47.53 \\
4900\end{array}$ & $\begin{array}{l}5.54 \\
5.52\end{array}$ & \multirow{2}{*}{-0.88} & \multirow{2}{*}{.189} \\
\hline \multirow{2}{*}{ INTE CRA CICN FAMILLAR } & $H D O$ & 39 & 21.94 & 2.50 & & \\
\hline & HEZA & 25 & 22.66 & 2.49 & $\cdot 0.96$ & .169 \\
\hline
\end{tabular}

(*) Significativo $\mathrm{P}<05$

( ) No significativo.

Cuadro $\mathrm{N}^{\circ}$ 3: Prueba t de student de la actitud por área de la madre frente al hijo y la hija

\begin{tabular}{|c|c|c|c|c|c|c|}
\hline AREA & GRIPO & $N$ & $\bar{X}$ & $D S$ & $t$ & $P$ \\
\hline CUID ADO YPROTECCION & $\begin{array}{l}F \square O \\
H O A A\end{array}$ & $\begin{array}{l}39 \\
15\end{array}$ & $\begin{array}{l}19.56 \\
19.99\end{array}$ & $\begin{array}{l}3.20 \\
5.02\end{array}$ & -0.19 & .425 \\
\hline $\begin{array}{c}\text { FEACEPCTCW Y ACEPTA DEL } \\
\text { FROBLENA }\end{array}$ & $\begin{array}{l}H D O \\
H D A\end{array}$ & $\begin{array}{l}39 \\
15\end{array}$ & $\begin{array}{l}30.67 \\
30.86\end{array}$ & 3.72 & -0.14 & .444 \\
\hline RELACICNES INTEREERS CNALES & $\begin{array}{l}H D O \\
H D D A\end{array}$ & $\begin{array}{l}39 \\
15\end{array}$ & $\begin{array}{l}20.77 \\
20.66\end{array}$ & $\begin{array}{l}302 \\
3.27\end{array}$ & 0.05 & .520 \\
\hline EXFECTATIVAS DE LOCRO & $\begin{array}{l}H \amalg O \\
H D O A\end{array}$ & $\begin{array}{l}39 \\
15\end{array}$ & $\begin{array}{l}26.48 \\
28.26\end{array}$ & $\begin{array}{l}3.92 \\
5.06\end{array}$ & -1.27 & .102 \\
\hline DISCIPLINA & $\begin{array}{l}H D O \\
H D A\end{array}$ & $\begin{array}{l}39 \\
15\end{array}$ & $\begin{array}{l}13.51 \\
14.20\end{array}$ & $\begin{array}{l}2.53 \\
4.16\end{array}$ & -0.60 & .274 \\
\hline PRE JUETCS & $\begin{array}{l}H D O \\
H D O A\end{array}$ & $\begin{array}{l}39 \\
15\end{array}$ & $\begin{array}{l}46.15 \\
47.38\end{array}$ & $\begin{array}{l}4.92 \\
9.79\end{array}$ & -0.49 & 330 \\
\hline INTE GRACICWN FARILLAR & $\begin{array}{l}H O O \\
H O A\end{array}$ & $\begin{array}{l}39 \\
15\end{array}$ & $\begin{array}{l}20.79 \\
21.50\end{array}$ & $\begin{array}{l}3.22 \\
5.18\end{array}$ & -0.56 & .288 \\
\hline
\end{tabular}

(*) Significativo $P<05$

( ) No significativo. 
Cuadro $\mathrm{N}^{\circ} 4$ : Prueba t de student de la actitud por área hacia el hijo del padre y la madre

\begin{tabular}{|c|c|c|c|c|c|c|}
\hline AREA & GRUPO & $N$ & $\bar{X}$ & $D S$ & $t$ & $P$ \\
\hline \multirow[b]{2}{*}{ CUIDADO Y PROTECCIÓN } & PADRE & 39 & 18.71 & 4.04 & \multirow{2}{*}{-2.25} & \multirow{2}{*}{.125} \\
\hline & MADDRE & 39 & 79. 66 & 320 & & \\
\hline \multirow{4}{*}{$\begin{array}{c}\text { PERCE PCTON Y ACEPTA DEL } \\
\text { PROBLE MA } \\
\text { RELACIONES } \\
\text { INTERPERSONALES }\end{array}$} & PADRE & 39 & 31.56 & 345 & \multirow{2}{*}{$\cdot 2.17$} & \multirow{2}{*}{879} \\
\hline & MADRE & 39 & 30.61 & 371 & & \\
\hline & $P A D R B$ & 39 & 20.66 & 2. $n$ & .007 & 472 \\
\hline & MADRE & 39 & 20.71 & 301 & & \multirow[b]{2}{*}{$.006 *$} \\
\hline \multirow[t]{2}{*}{ EXPECTATIVAS DE LOGRO } & $\begin{array}{l}P A D R B \\
M A D R E\end{array}$ & $\begin{array}{l}39 \\
39\end{array}$ & $\begin{array}{l}28.79 \\
26.43\end{array}$ & $\begin{array}{l}4.33 \\
3.92\end{array}$ & \multirow[t]{2}{*}{2.53} & \\
\hline & PADRE & 39 & $\begin{array}{l}26.43 \\
12.58\end{array}$ & $\begin{array}{l}3.92 \\
2.98\end{array}$ & & \multirow{2}{*}{.067} \\
\hline DISCIPLINA & $\triangle A A D R E$ & 39 & 1351 & 2.53 & .250 & \\
\hline \multirow{2}{*}{ PREJUICIOS } & $P A D R B$ & 39 & 47.53 & 5.54 & \multirow{2}{*}{ 2.16 } & \multirow{2}{*}{877} \\
\hline & $M A D R E$ & 39 & 46.15 & 4.92 & & \\
\hline INTE GRA CIOAN FAMILIAR & $\begin{array}{l}\text { PADRE } \\
\text { MADPE }\end{array}$ & $\begin{array}{l}39 \\
39\end{array}$ & $\begin{array}{l}21.94 \\
20.79\end{array}$ & $\begin{array}{l}2.50 \\
3.22\end{array}$ & 2.76 & $.039 *$ \\
\hline
\end{tabular}

CUADRO N ${ }^{\circ}$

PRUEBA t DE STUDENT DE LA ACTITUD POR ÁREAS HACIA LA HIJA DEL PADRE Y LA MADRE

\begin{tabular}{|c|c|c|c|c|c|c|}
\hline AREA & GRUPO & $N$ & $\bar{X}$ & DS & $t$ & $p$ \\
\hline CUIDADO Y PROTECCION & $\begin{array}{l}\text { PADRE } \\
\text { MADRE }\end{array}$ & $\begin{array}{l}15 \\
15\end{array}$ & $\begin{array}{l}19.13 \\
19.93\end{array}$ & $\begin{array}{l}4.01 \\
5.02\end{array}$ & $-0,48$ & .316 \\
\hline PERCEPCION Y ACEPTA DEL & PADRE & 15 & 32.40 & 3.11 & 0,70 & \\
\hline PROBLEMA & MADRE & 15 & 30.86 & 7.85 & & .728 \\
\hline $\begin{array}{l}\text { RELACIONES } \\
\text { INTERPERSONALES }\end{array}$ & $\begin{array}{l}\text { PADRE } \\
\text { MADRE }\end{array}$ & $\begin{array}{l}15 \\
15\end{array}$ & $\begin{array}{l}20.93 \\
20.60\end{array}$ & $\begin{array}{l}2.46 \\
3.27\end{array}$ & 0.25 & .599 \\
\hline EXPECTATIVAS DE LOGRO & $\begin{array}{l}\text { PADRE } \\
\text { MADRE }\end{array}$ & $\begin{array}{l}15 \\
15\end{array}$ & $\begin{array}{l}28.13 \\
28.26\end{array}$ & $\begin{array}{l}4.35 \\
5.06\end{array}$ & -0.07 & .472 \\
\hline DISCIPLINA & $\begin{array}{l}\text { PADRE } \\
\text { MADRE }\end{array}$ & 15 & $\begin{array}{l}13.26 \\
14.20\end{array}$ & $\begin{array}{l}2.66 \\
4.16\end{array}$ & -0.74 & .230 \\
\hline PREJUICIOS & $\begin{array}{l}\text { PADRE } \\
\text { MADRE }\end{array}$ & $\begin{array}{l}15 \\
15\end{array}$ & $\begin{array}{l}49,00 \\
47.33\end{array}$ & $\begin{array}{l}5.52 \\
9.79\end{array}$ & 0.57 & .716 \\
\hline INTEGRACION FAMILIAR & $\begin{array}{l}\text { PADRE } \\
\text { MADRE }\end{array}$ & $\begin{array}{l}15 \\
15\end{array}$ & $\begin{array}{l}12.66 \\
21.60\end{array}$ & $\begin{array}{l}2.49 \\
5.18\end{array}$ & 0.71 & .761 \\
\hline
\end{tabular}

*) Significativo $\mathrm{P}<\mathrm{OS}$

( ) No significativo.

\section{Conclusiones}

1. Se acepta la hipótesis nula que nos indica que tanto el padre como la madre no difieren en sus actividades generales hacia el hijo y la hija con retardo mental leve.

2. Los padres no difieren de las madres en sus actitudes hacia el Cuidado y Protección, tanto del hijo como de la hija con retardo mental leve. En ambos casos las actitudes promedio corresponden a la categoría de ambivalencia actitudinal o de actitud indefinida.

3. Los padres no difieren de las madres en sus actitudes hacia la Percepción y aceptación del problema tanto del hijo como de la hija con Retardo mental leve.

4. Los padres no difieren de las madres en sus actitudes hacia el Prejuicio tanto del 
hijo como de la hija con Retardo mental leve. En ambos casos las actitudes promedio corresponden a la categoría de positiva.

5. Los padres no difieren de las madres en sus actitudes hacia la integración familiar tanto del hijo como de la hija con retardo mental leve. En ambos casos las actitudes promedio corresponden a la categoría de tendencia a actitudes positivas.

\section{RECOMENDACIONES}

1. Incrementar el nivel informativo de los padres en relación al retardo mental y orientar su enfoque hacia la comprensión de las necesidades, las actitudes y las capacidades especiales de la familia del niño con Retardo mental, preparándolos y estimulándolos para participar activamente en el proceso de educación, formación y rehabilitación del niño deficiente.

2. Se espera que en investigaciones futuras se realice el estudio de las actitudes de los padres frente al retardo de sus hijos, tomando en cuenta la comparación de estos en grupos de diferente status socioeconómico.

3. Fomentar que el Ministerio de Educación a través de la dirección de Educación Especial incluya dentro de los programas de escuela de padres temas que aborden la formación de actitudes con respecto a los hijos incidiendo en combatir los prejuicios que existen con respecto al educando excepcional, no solamente en los padres sino también en todo el grupo familiar. 


\section{REFERENCIAS BIBLIOGRÁFICAS}

Ackerman, N. W. (1982). Diagnóstico y tratamiento de Las relaciones familiares. Buenos Aires: Editorial Paidos.

Bach, H. (1980). La deficiencia mental: Aspectos pedagógicos. Madrid: Editorial Cincel S.A.

Brauner y Brauner (1981). La educación de un niño deficiente mental. España: Editorial Gráfica Ema.

Edgerton, R. (1980). Retraso mental. Madrid: Ediciones Morata S.A.

Gisbert, M.; Cabada, M.; y Otros. (1984). Educación Especial. España: Editorial Cincel S.A.

Hermoza, A. (1986). Estudio comparativo de Actitudes de un grupo de madres de niños con Retardo mental Fronterizo con otro grupo de madres cuyos niños presentan Retardo mental Leve. Lima - Perú: Tesis para optar el título de Licenciado en Psicología U.P.S.M.P.

Munné, F. (1980). Psicología Social. España: Ediciones CEAC.

Paucar, C. (1990). Actitudes maternas frente a sus hijos con Retardo mental que asisten al Centro de Educación Estatal \# 01. Lima - Perú: Tesis para optar el título de Licenciado en Psicología U.P.S.M.P.

Quispe, G. (1990). Actitudes maternas hacia sus hijos que presentan retardo mental por diferente etiología. Lima - Perú: Tesis para optar el título de Licenciado en Psicología U.P.S.M.P.

Ruiz, C. (1986). Escuela para padres en Educación Especial. Lima - Perú: Ministerio de Educación, Dir. Gen. de Educación Inicial Especial.

Sumers, G.F. (1978). Medición de actitudes. México: Editorial Trillas.

Sotil, A. (1987). Desarrollo social y personal de un grupo de jóvenes con Retardo mental a través de un programa integral de capacitación laboral. Lima - Perú: Tesis para optar el grado de Doctor en Letra especialidad Psicología U.N.M.S.M.

Ugarriza, N. (1987). Modelo de interferencias causales de las actitudes de las madres hacia el hijo con Retardo mental. Lima Perú: Tesis para optar el grado de Doctor en Letra especialidad Psicología D.N.M.S.M.

Heward, W. (2000). Niños Especiales. España: Editorial Prentice Hall. 